of long period, about 900 years, with coefficient about $4^{\prime \prime}$, attributable to an error in Hill's value of the great inequality. Solutions by the method of least squares give a correction to the mean longitude of Hill's tables

$$
\begin{aligned}
-4 " .44+0 \text { o.09 } T+2 " .84 & \sin \left(5 g-2 g^{\prime}\right) \\
& +22^{\prime \prime} .78 \cos \left(5 g-2 g^{\prime}\right),
\end{aligned}
$$

where $T$ is the time in centuries after $1850, g$ is the mean anomaly of Saturn, and $g^{\prime}$ that of Jupiter. After removing these terms from the differences, another solution for the secular terms gives a correction to the true longitude of Hill's tables

$$
\begin{array}{r}
- \text { o."03 } \sin g+\text { o". } 47 \cos g-\text { o". } 57 T \sin g \\
+ \text { o".06 } T \cos g-\text { o.. } 25 T^{2} \sin g \\
\quad+\text { o". I I } T^{2} \cos g .
\end{array}
$$

In both of the expressions above, the first two terms are without significance, so far as errors in Hill's tables are concerned ; they simply represent the elements of the orbit that must be adjusted simultaneously with solutions for the other terms.

When these additional terms are removed, the residuals still show strongly systematic trends, reaching $\mathrm{I}^{\prime \prime}$, which so far have not been amenable to analysis. The reality of the long-period and secular terms cannot be doubted, but it is possible that the solution for the secular terms has been affected by the presence of other terms of similar period.

The curve for the error in the radius vector is strongly periodic, with semiamplitude about 0.00005 astronomical units. The errors cannot be connected with a single periodic term in Hill's theory; they must be of a more complicated nature. The curve for the error in the latitude also shows evidence of periodicity. The size of the errors is much smaller than for the other two coordinates, the average difference without regard to sign being o". Io. It is probable that they are associated with errors in Hill's secular perturbations. The small size of the errors in the latitude must be associated with the relatively small size of the latitude perturbations themselves, which results from the disturbing planets being nearly in the plane of Saturn's orbit, and they constitute strong external evidence that no gross error has been committed in the numerical integration.

The general conclusion is that Hill's tables of Saturn contain important errors, which increase with the time, reaching $6^{\prime \prime}$ during the next century. The cause is not completely known; to ascertain it would probably involve the repetition of much of Hill's work.

This work was supported by the Office of Naval Research.

U. S. Naval Observatory, Washington, D. C.

Climenhaga, J. L., A. McKellar, and R. M. Petrie. Spectrographic observations of $\varepsilon$ Ursae Minoris.

Sixty-five spectrograms of $\epsilon$ Ursae Minoris have been obtained with single-prism, moderate dispersion and several with lower dispersion in the ultraviolet. The purpose was to examine the spectrum of this G-type single-line binary star with respect to phase, in order to seek evidence of spectral peculiarities possibly associated with the very peculiar light curve obtained by Guthnick.

It was noted that emission components occur in the centers of the $\mathrm{H}$ and $\mathrm{K}$ lines of $\mathrm{CaII}$. These emission lines showed no variations in intensity and their radial velocities followed the radial-velocity curve for the absorption lines. No spectral peculiarities nor changes with phase were detected to support Guthnick's hypothesis of a gaseous ring associated with the system. Evidence of the presence of an earlier-type companion was sought, but not found, in the spectrum in the ultra-violet as far as $\lambda 3440$. It is suggested that further photoelectric observations of the star should be made.

A new set of spectrographic orbital elements was derived for $\epsilon$ Ursae Minoris. They were in substantial agreement with the former set obtained by J. S. Plaskett in I9I0. The work will be given in detail in Pub. Dom. Astroph. Obs. 8, No. 14.

Dominion Astrophysical Laboratory, Victoria, B. C.

\section{Cuffey, James. A linearity test for photoelectric equipment.}

Photoelectric photometers, which use photomultiplier cells, direct current amplification, and recording meters of the Esterline-Angus or Brown Electronik type, are sometimes assumed to be strictly linear devices. It is, however, desirable to have a convenient and accurate means for testing the equipment as a working unit.

There are two aspects to the problem; the first requires a test for linearity over the limited intensity range corresponding to a single full-scale deflection of the recording meter; and the second 